Volume 12 | Issue 2

June 2021

\title{
The Power of Connections: How a Novel Canadian Men's Wellness Program is Improving the Health and Well-Being of Indigenous and Non-Indigenous Men
}

\author{
Iloradanon Efimoff ${ }^{*}$ \\ University of Manitoba, Canada, efimoffi@myumanitoba.ca \\ Lyana Patrick* \\ Simon Fraser University, Canada, lyana_patrick@SFU.ca \\ Viviane Josewski \\ University of British Columbia, Okanagan, Canada, viviane.josewski@ubc.ca \\ Paul Gross \\ University of British Columbia, Canada, paul@dudesclub.ca \\ Sandy Lambert \\ DUDES Club Elder and External Liaison, Canada, sandy@dudesclub.ca \\ Victoria Smye \\ Western University, Canada, vsmye@uwo.ca
}

Recommended Citation

Efimoff, I., Patrick, L., Josewski, V., Gross, P., Lambert, S., \& Smye, V. (2021). The power of connections: How a novel Canadian men's wellness program is improving the health and well-being of Indigenous and non-Indigenous men? The International Indigenous Policy Journal, 12(2). https://10.18584/iipj.2021.12.2.10896 


\title{
The Power of Connections: How a Novel Canadian Men's Wellness Program is Improving the Health and Well-Being of Indigenous and Non-Indigenous Men
}

\begin{abstract}
The DUDES Club is a novel men's health and wellness organization founded in 2010 in Vancouver, BC. Since 2017, the DUDES Club has rapidly expanded in Northern BC and, with the help of a partnership with the First Nations Health Authority, has grown to include 40 sites in British Columbia and 2 sites nationally. In this study, we analyze data from interviews $(n=5)$ and 15 focus groups $(n=101)$ conducted as part of a program evaluation with DUDES Club members, Elders, providers, and health care professionals. We focus on men's experiences with the DUDES Club to identify four main themes in the data: brotherhood and community, accessible health care information, disrupting colonial constructions of masculinity, and systemic and structural challenges. We conclude with policy recommendations.
\end{abstract}

\section{Keywords}

Indigenous men, community-based health promotion programming, men's health, qualitative program evaluation, social support

\section{Author Note}

*These authors contributed equally to this work.

\section{Acknowledgments}

We would like to dedicate this article to the late Mr. Richard Johnson and the late Mr. Henry Charles. Mr. Richard Johnson co-founded the DUDES Club and was an outreach worker for the Positive Outlook Program at Vancouver Native Health Society. He laid the strong foundation on which the DUDES Club was built. Henry Charles was our Elder from 2011 to 2017 and joined the spirit world on a very sad and tragic rainy Friday night in late January 2017. He was a source of comfort, culture, humour, and knowledge for all members.

\section{Creative Commons License (2) $\odot \Theta \Theta$}

This work is licensed under a Creative Commons Attribution-Noncommercial-No Derivative Works 4.0 License. 


\section{The Power of Connections: How a Novel Canadian Men's Wellness Program is Improving the Health and Well-Being of Indigenous and Non-Indigenous Men}

Every two weeks in Vancouver's Downtown Eastside (DTES) neighbourhood, a group of men gather together for a few hours in the evening to share food and ideas, to learn and have fun, and to take a break from their often-difficult daily realities. This unlikely gathering started 10 years ago when frontline workers recognized the need for a safe and supportive space for men to gather. What started as a support group organized around the Positive Living Outreach Program at the Vancouver Native Health Society has blossomed into a national model for men's health and well-being. This group is called the DUDES ${ }^{1}$ Club.

Indigenous Peoples in Canada experience a disproportionate burden of both mental and physical health issues (Nelson \& Wilson, 2017; Rotenberg, 2016). These health inequities must be understood in light of colonialist and neo-colonialist practices (such as residential schools, the Sixties Scoop, and the overrepresentation of Indigenous children in care), widespread poverty, homelessness, and racism (Currie et al., 2012; Patrick, 2014; Press, 2019; Spence et al., 2016). In healing from past and ongoing effects of colonization, culture is a key source of strength for many Indigenous communities (Assembly of First Nations \& Health Canada, 2015; Gone, 2013). An emerging body of evidence for "culture as treatment" points to the significance of interconnectedness in healing and underscores the need to incorporate Indigenous cultural practices into health programming (Barker et al., 2017).

Generally, men are less likely to seek medical attention with regards to their mental well-being. This tendency can have tragic consequences: Men are 3 times more likely to die by suicide than women (Goldenberg, 2014; Statistics Canada, 2019). Indigenous men are at an even higher risk for suicide (Kumar \& Tjepkema, 2019). Men's high rates of suicide might, in part, be due to inadequate diagnosis and treatment of men's mental health issues (Oliffe et al., 2010; Wide et al., 2011). To this end, the American Psychological Association issued its first guidelines for practice with men and boys (Pappas, 2019). Conformity to dominant ideas of masculinity that stress male independence and fearlessness ("the strong silent type") may further lead men to disregard their health and avoid seeking timely health care (Goldenberg, 2014, p. S142), which is a concern that is particularly pressing for older men (Tannenbaum \& Frank, 2011).

Despite the disproportionate burden of poor health outcomes in Indigenous communities and the low likelihood of men seeking medical care, there is a dearth of research on Indigenous men's health services in Canada (e.g., Hackett et al., 2021). Internationally, Men's Sheds are a successful men's health promotion program, with research indicating that effective health programming for men has unique features (Wilson \& Cordier, 2013). However, more research is needed to fully and rigorously evaluate Men's Sheds (Milligan et al., 2016). One important factor is peer support. Peer support groups can increase social support, resilience (Robinson et al., 2015), and health (Heisler et al., 2010; Richmond et al., 2007).

The DUDES Club, founded in British Columbia, Canada, in 2010, is another promising men's health promotion program with an Indigenous focus. In 2016, a quantitative evaluation of the DUDES Club showcased the club's ability to promote overall well-being through culturally safe health services (Gross

${ }^{1}$ DUDES is an acronym for Dudes United Defending Equality and Solidarity. 
et al., 2016): Compared to infrequent attenders, frequent attenders experienced improved quality of life, mental health, physical health, health confidence, peer support, spiritual wellness, and social support. Furthermore, compared to non-Indigenous members, Indigenous members felt more trust and support with the group and felt that the Club helped them connect to their cultural heritage (Gross et al., 2016).

The purpose of this study is to investigate Indigenous and non-Indigenous men's experiences with the DUDES Club and how the model could be extended to other communities. We conclude with five policy recommendations for those interested in creating their own DUDES Club. In this article, we discuss interconnecting and mutually informative themes identified in our qualitative findings from our evaluation of the DUDES Club.

\section{Program Background: How the DUDES Club Began}

The DUDES Club is a grassroots initiative that formed in 2010 in response to community concerns about the relative lack of men's health programming in the DTES of Vancouver. The DTES forms part of the Traditional Territories of the Squamish, Tsleil-Waututh, and Musqueam First Nations. As one of Vancouver's earliest neighbourhoods, the DTES was a vibrant working-class area that provided a social, cultural, and retail hub for the growing city. In the 1980s, intersecting factors contributed to increasing social problems including homelessness, an open drug market, poverty, crime, and mental illness. These factors disproportionately impact Indigenous people who in 2016 made up 2.2\% of the population in Vancouver, but $14 \%$ in some areas of the DTES (Huang, 2019). In 2013, two pilot sites were established (one in Smithers and one in Prince George, BC). To date, there are 40 partner sites throughout BC (DUDES Club, n.d.). The objectives of the Club are threefold: (a) to build solidarity, brotherhood, trust, and positive role models together with men; (b) to promote holistic, cost-effective men's health through accessible dialogue, education, and health screening opportunities; and (c) to enable men to regain a sense of pride, purpose, and fulfillment in their lives. In carrying out these objectives, the Club adheres to the values of holistic health, inclusivity, and non-judgment (anyone identifying as male is welcome). The club's motto, "Leave your armour at the door," sets the groundwork: Men may wear "armour" to protect themselves on the street, but in the DUDES Club, there is no need for it. The men share openly with each other on a variety of topics, ranging from diet to sexual abuse. Members are always welcome regardless of their physical or mental state as long as they are respectful during the gatherings.

The three different clubs discussed in this article are held in a cultural drop-in centre, an HIV outreach office, and alongside a health clinic. The Vancouver site hosts 50 to 60 members regularly. In contrast, the gatherings at the pilot sites are smaller with 10 to 12 men attending each meeting. Each site has an Indigenous framework, which includes Elders, the Medicine Wheel, and health care practitioners with cultural safety training, in order to provide a culturally safe space for the mostly Indigenous members attending the DUDES Club. Sites routinely incorporate bonding activities, such as bingo or ice fishing. All sites share a meal and engage men in a health discussion. If a health care provider is present, it may be a technical conversation; if only the members and the Elders are present, discussions are much more anecdotal and personal. Some club members are extensively involved in governing the Club through "think tank meetings," planning activities, meals, and discussion topics. Those who formally volunteer at the Club receive modest financial compensation for their time. 


\section{Method}

\section{Indigenous and Critical Theoretical Perspectives}

This research was informed by Indigenous and critical theoretical perspectives (e.g., Battiste, 2009; Collins, 2015; Innes \& Anderson, 2015) that emphasize Indigenous voices and narratives and draw attention to the intersecting contexts that shape men's experiences of health and health care. Thus positioned, we use intersectionality as an analytical lens for gaining a rich and complex understanding of how and why the DUDES Club improved men's quality of life, mental health, and physical health.

\section{Data Collection}

This article draws on data from 15 focus groups $(n=101)^{2}$ and five individual interviews with club members, Elders, providers (e.g., site managers), and health care professionals (e.g., doctors and nurses at the Club). We conducted six focus groups and three individual interviews at the Vancouver site, three focus groups and one individual interview at the Smithers site, four focus groups and one individual interview at the Prince George site, and two focus groups at a gathering of all three sites. There were 3 to 10 participants at each focus group and a few participants attended more than one focus group. We interviewed providers and club members separately from focus group members to avoid any influence on member input. Overall, we include data collected from approximately 30 club members, four Elders, one provider, and six health care professionals. All of the focus groups and interviews but one were audio-recorded (one interviewee opted not to be recorded). Focus groups lasted between approximately two and two-and-a-half hours, and individual interviews ranged from approximately 15 to 60 minutes. We used semi-structured interviews with a set of prompt questions to guide each focus group and interview. Our questions focused on the men's experiences in the DUDES Club (both positive and negative), how the Club impacted their health and well-being, how the Club compared to other local groups, and how to improve the Club and their experience. All focus group members were asked for their input, and all participants received a $\$ 30.00$ honorarium. Our research procedures were approved by the University of British Columbia Research Ethics Board.

All the men in our study came from a context of disenfranchisement, such as unstable housing, unemployment, and poverty (Gross et al., 2016), and many lived with complex histories of trauma, mental health, and/or substance use issues, as well as other comorbid conditions. For example, based on a survey conducted at the Vancouver site by Gross et al. (2016), more than $80 \%$ of men reported living in the DTES of Vancouver, a neighbourhood widely known as one of the poorest neighbourhoods in North America, and more than 60\% reported experiencing unstable housing. The majority of participants in this survey self-identified as Indigenous (63.3\%) and most reported being in their middle years of life. Lastly, although almost $75 \%$ of participants reported being single at the time of the study, nearly $60 \%$ had children, but fewer than $5 \%$ lived with their children. Notably, these characteristics only describe the membership at the Vancouver site, as this demographic information was collected as part of the larger program evaluation survey.

\footnotetext{
${ }^{2}$ We could not obtain the exact number of participants for four of the focus groups due to data recording errors. We used the average number of participants in the 11 focus groups where we had the exact number of participants to estimate the number of participants for these four focus groups. We then added these four estimated values to the actual values to calculate the $n$.
} 


\section{Data Analysis}

We undertook an interpretive thematic analysis using procedures described in the literature for qualitatively derived data (Thorne, 2016). We began by transcribing all audio recordings of the interviews. Once transcribed, we cleaned and anonymized the transcripts (to correct any transcriber errors and protect participant and organizational privacy) and then uploaded them into NVivo (qualitative analysis software). Our team, consisting of Indigenous and non-Indigenous members, then analyzed the data in a multi-step process. First, we looked at the data inductively, systematically, and reflectively, reading and re-reading the transcripts and discussing initial impressions and ideas. Second, we independently coded all transcripts based on an evolving coding structure. The purpose of the coding was to identify key concepts that we noted in the data, as well as conceptual linkages and patterns of relationships. Throughout the coding process, we created, combined, and discarded codes in an iterative process to further refine the codes. This involved creating hierarchical models through NVivo to see the codes from a macro perspective and concept maps to further identify connections between codes. As our coding became less descriptive and more interpretive, we began to reflect on the theoretical constructs behind key codes by drawing on our theoretical perspectives and making linkages between the literature and the data.

Specifically, we drew on intersectionality as an analytical lens for examining and developing a better understanding of how becoming members of the DUDES Club shaped Indigenous men's experiences of health and well-being. We situated participants' experiences within the intersecting contexts of past and ongoing colonialism, dominant gender norms, poverty, racism, class, and other forms of stigma and discrimination. Furthermore, Indigenous Elders Mr. Sandy Lambert and the late Mr. Henry Charles were key members of the research team, guiding our analysis by speaking to what they thought were the most important aspects of the DUDES Club, based on their years of experience with the group. This helped focus our analysis and ensure that our conclusions were grounded in Indigenous perspectives.

Through this analysis and our research team's years of experience with the DUDES Club, we created the policy recommendations at the end of this article. We based these policy recommendations on members' experiences and our perceptions of what was most effective in facilitating the creation of a DUDES Club.

\section{Results}

Club members, Elders, providers, and health care professionals shared detailed accounts of their experiences with the DUDES Club. In this section, we present four themes that we identified in participants' stories: a feeling of brotherhood and community, accessible health care information, disruptions of colonial masculinities, and the systemic and structural challenges the Club helped participants face.

\section{Feeling of Brotherhood and Community}

One of the largest themes identified in all focus groups and interviews was feeling a sense of brotherhood and community. The members, Elders, service providers, and health care professionals all described this as one of the most powerful and effective aspects of the Club. One way the Club seemed to create feelings of brotherhood and community is by making the members comfortable enough to share their 
experiences. There was consensus among the men that members respect each other in part by respecting the rules of confidentiality. As such, several DUDES Club members described how they openly shared many emotionally sensitive parts of their histories, including residential school experiences, sexual abuse, anger issues, mental health concerns like post-traumatic stress disorder (PTSD) and suicidal ideation, historical trauma, loneliness, and experiences with HIV. For example, one member described:

We've had issues discussing like big issues, prostate, we've had issues like male sexual abuse where people actually came out in the room and said they had been abused. Men don't talk about this to other men. ... They feel safe enough here they can, this is a safe haven. It's a sanctuary they can come in, it doesn't matter what that medical problem is, discuss it and we don't talk about it on the street. Because if it does the person who takes it out is no longer coming here.

The fact that some members were able to share these types of stories highlights the safe and trusting relationship they had with each other. While such relationships take time to develop, a similar atmosphere of safety and trust is characteristic in each of the clubs. The safe space created at DUDES Club gatherings seems to allow some members to be vulnerable: to open up and share stories, cry, ask for help, discuss mental health issues, and simply be comfortable in a safe space.

Some members also explained that they felt comfortable in the group because a non-judgmental atmosphere was maintained, and because the group actively attempts to decrease the stigma around the men involved in the Club. One group challenged stereotypes about their members by playing horseshoes in public with friends. An Elder explained that the presence of celebrities in the Club (such as famous football players) helped to de-stigmatize low-income areas. As one of the members said:

You can come in here and even if you are using drugs you talk about all kinds of stuff, it's nonjudgmental. That's the big thing, because you walk into most places and I'm an addict and this is a problem.

Part of the non-judgmental atmosphere is maintained because it is a men-only club, as one member explained:

A place to feel safe to share in, issues that, you know, we confront. As a man it might be different than what women would confront.

This sense of brotherhood and community seemed to be connected to the sense of ownership some members had around their clubs. Through the creation of their own club, in their own space, with their own rules, many of the members described an increased sense of personal empowerment and agency. Members routinely decide on speakers, topics, activities, and food for meetings. Discussing issues and making these decisions together seems to create a sense of community felt by many of the men, as well as a sense of solidarity around their experiences with the DUDES Club and as men in general. This sense of ownership and self-determination over the Club appeared to lead to an increased sense of pride in some men, which, in turn, seemed to allow for greater solidarity and brotherhood. Furthermore, many of the members volunteer for their clubs, describing a sense of purpose and meaningful occupation, which seemed to increase their self-worth and self-esteem, further investing them in the program. These positives associations are, in part, where members' sense of "brotherhood" came from: 
The majority of my time spent here is volunteering so it gives me a sense of purpose so I feel like I did a good job and I'm good to people and later on they remember that and they come up to me later on and say, "Hey dude."

Some DUDES Club members even took it upon themselves to engage the community in their activities, building a wider community for members. They described seeking out lawyers, town councillors, church members, legal aides, politicians, and people they met on the street to get involved with the DUDES $\mathrm{Club}$ - and they were often successful. Some members attended town meetings with council members to voice concerns, connect with politicians, and brainstorm ways to connect with the community to showcase the Club and share resources. A few members have even talked about different public relations opportunities and were actively involved in a Globe and Mail article about the DUDES Club (Barton, 2016). The sense of ownership over the Club, our findings suggest, solidifies feelings of brotherhood and community, which in turn encourages members to work together to improve their club and experiences.

In neighbourhoods where the focus groups were conducted, men-often Indigenous men-appeared to feel especially disempowered by the structural inequities and violence they faced daily. In the flattened hierarchy of the DUDES Club, one member described how many members found a place where their voice mattered:

You know I get the sense that for a lot of men, I think it - they come to the DUDES Club, I think to finally get a better picture just how important men are in our society, especially in the society of the Downtown Eastside. That our voice is just as important as anybody else's, you know, we all have a voice and it gets heard at the DUDES Club, you know, everybody is willing to listen and start acting on what our participants share, okay.

The sense of brotherhood and community was linked to some members' discussions of the importance of strength in numbers. Members sometimes used their club and their connections to advocate for themselves and others. Attendance at the DUDES Club appeared to help many members understand that their voice was as important as any other voice in society. Some explained that their voices were often not heard in other arenas and it was empowering to have their voices heard at the DUDES Club. Beyond advocacy, members, Elders, providers, and health care workers discussed being strong together as peer support:

Member: Just to chat and be friendly, get to know people-if you're running through people downtown you know they're with the men's club or whatever, and if you get in trouble, you know the men you run into-they will help you out in other situations - it's just mostly the friendship.

Elder: And if they're drugged, under the influence of drugs or anything, everybody knows to, they watch for each other. Basically what did I say, [word in Musqueam language] means to pick up somebody that's fallen, right?

Health care professional: I could see DUDES Clubs popping up all over, and not necessarily talking about men's health, but being a place of community and a place of brotherhood and a place of joy and a place of sadness, and a place of bonding ... after I watched the [DUDES Club 
documentary], I said, "Wow, that kind of healing is so far beyond what I'm offering on a medical model.”... I can help people with their medical stuff in my 10 minutes. ... But I can't do that ... that connection with other men and people.... It's just really compelling.

Some men also discussed the ways in which providing peer support was empowering. It provides a sense of purpose as well as building community. Peer support also seemed to help many members feel less lonely and more connected to other people. Some discussed being able to ask for help when they need it. In fact, many men indicated that they attended the DUDES Club because of the friendship and community they experienced. The support gained from other men is a vital component of men's health in general and, as indicated by the health care professional's quote above, health care professionals cannot help with social connection. Furthermore, some members discussed how the connection with other people at the DUDES Club reduced their stress levels, as they socialized and sought sanctuary from their "street identity." Thus, the DUDES Club provides a safe and accepting social environment, which aids in the improvement of members' health and wellness.

The cultural and personal senses of identity and resilience as well as the individual healing journeys of DUDES Club members were important outcomes, which are related to their shared experiences of brotherhood and community. For example, one member described his journey through mental illness and overcoming barriers to being in the community and around others. His words revealed how the DUDES Club helped him build resiliency:

Being in a large group, building confidence and overcoming anxiety and stuff like that. That's the-I get a lot out of DUDES Club-like, you know it's helped me to be-to overcome anxiety and being in a large group.

Many members discussed how challenges they face on the street, such as the inaccessibility of appropriate services and concerns about comfort and safety, occasionally prevented them from attending programs, including the DUDES Club. By establishing a support network and structure, the DUDES Club has helped to create a sense of safety in the community. This sense of safety has facilitated participation because feeling unsafe prevents many men in these communities from moving and travelling at certain times. Therefore, this fellowship creates long-term connections, fosters a sense of safety on the streets, and encourages resiliency in how some members face day-to-day challenges. One member described how this created an essential lifeline of information in what, for some, felt like a vacuum:

Amongst one another we can talk, we can explore, we can see if other people suffer the same things, you can share ideas-perhaps somebody did suffer that and they can let us know how they overcame it.

Many members also described how resiliency was built through ongoing participation in the Club. They extended the values nurtured and promoted within the group onto the street where they could put the things they had learned into action. One member explained:

Part of the reason I come [to the DUDES Club] is for friendship, but also there's problems on the street that for me ... if they see me and there's a problem, they will help me out, because ... there's some places where you go downtown aren't safe. 
Lastly, the presence of Elders and others to guide, build confidence, and provide support for the members was a vital aspect of the DUDES Club and a key component in developing cultural identity, particularly for Indigenous men. For example, Indigenous members discussed the value of Elders speaking their language and sharing cultural teachings. One explained that the DUDES Club was a good way to connect with Indigenous culture and that most people have to go to prison to find that cultural connection. By having Indigenous Elders and ensuring Indigenous cultural teachings are central, the Club provides another avenue for Indigenous men to connect with their culture.

Owing to the club's men-only and non-judgmental atmosphere, and the peer support provided, many members felt comfortable in building authentic relationships with other club members. Members' sense of ownership over the Club contributed to feelings of responsibility and engagement. This, in turn, reinforced the sense of brotherhood and community. This experience of brotherhood, community, and ownership further facilitated resiliency, healing, and identity development within members. In addition, the DUDES Club also facilitated observable health changes, as we will discuss next.

\section{Accessible Health Care Information Facilitates Reconnection to Body and Mind}

The majority of DUDES Club members are above 40 years of age (Gross et al., 2016). In one focus group, providers explained that, when men were young, they could ignore the doctor and feel invincible; however, as they approach age 50, they become more health conscious. Some members explained that they attended the Club because men's health information is more important as men age. However, accessing health care was not always easy. Members shared stories of avoiding medical care because of poor treatment, such as being denied painkillers because they were stereotyped as being addicts, being hung up on by 911 operators, and feeling rushed in the doctor's office. Some reported receiving better treatment with an advocate present. Furthermore, some were unable to seek health care because of accessibility issues. For example, some members did not have a vehicle to attend doctor's appointments. Poor treatment, accessibility issues, and constructions of masculinity have made many of the men in the group very reluctant to seek health care services, even when urgently needed.

The DUDES Club provides highly accessible, low-barrier care: The men do not have to sit in a waiting room and feel rushed when seeing a doctor. They appear relaxed and comfortable, ready to learn about their health. They are allowed to participate if intoxicated, as long as they are not disrespectful. Many members described the DUDES Club health care providers as approachable, and there is no hierarchy; the men all call each other by their first name (including the doctor and lawyer). As one member explained:

When we go in there we're equals. Got nurses, they show up. [Name of Elder] shows up. [Name of Elder] shows up, you show up, it doesn't matter. We're all the same. It's not an us and them thing.

Some of the members turned to the DUDES Club for help when typical health care settings were unsuccessful. Nurses have noted that some men who would not come into the clinic would still attend the DUDES Club for health information. A health care professional explained:

An example is a patient that had a lot of conflict coming to the clinic, because of ... many factors ... it just wouldn't work well sticking with the typical appointments and waiting because it would 
lead to escalated behaviour in the waiting room ... his needs not being met. ... I stopped providing care for him. But he still found sort of that fraternity in the DUDES Club. ... I feel like the DUDES Club was almost like a safety net in some way.

The health information provided at DUDES Club is also accessible. The members explained that the health care professionals welcomed any questions and explained things clearly. This is evidenced by the fact that members recited health advice during the focus groups:

Yes he tells us about medication, what's new, what's coming out and what to take and don't go take the one because it will give you nightmares there are better ones he says. So for HIV he gives us that, he tells us everything about the medication when it comes in and then what new ones are coming out.

The accessibility of health information is broadened because the information does not remain only in the group. Many members of the DUDES Club share this information and have pointed out that their knowledge may "save a life." One particularly salient example was told by a health care professional:

I had a really young guy, who was in his early to mid-twenties ... he's vomiting blood. So he's already got a lot of damage done to his liver and he's talked about, "Yeah, there's these guys out in the community and they've got these DUDES shirts and it's like some sort of DUDES Club thing. ... Yeah a few of them have talked to me," and I'm like, "Oh yeah, I know some of those guys." So they are getting out, they are talking to people in the community.... So the information is going beyond the group and getting out into the community.

The physical location of the DUDES Clubs is also important for accessibility. Two of the sites are welllocated, and one site has a slightly less desirable location. Bus schedules tie into this accessibility, with some members living in more remote locations missing meetings due to inconsistent or non-existent bus service.

Overall, many members expressed their satisfaction with the health care they received at the DUDES Club. Many of the men considered the doctor at the DUDES Club their family doctor and this seemed to increase the likelihood of them seeking health care services. One health care professional explained that the DUDES Club was the preferred means of accessing health care services for many men. The members appeared happy with the DUDES Club doctor:

[The doctor] explains everything to everyone ... the doctor is a really great doctor like you can ask any question about any disease and he tells you the best way to deal with it.

In particular, it is important for health care professionals involved in the Clubs to understand the members' experiences. For example, one member explained that it was important to have a doctor who was aware of health issues related to their neighbourhood. Others indicated the importance of being able to voice their concerns about "housing, health, [and] support."

The outcomes of such accessible health care are evident in members' reconnections to their body and mind. The men in this study shared many stories highlighting how the DUDES Club facilitated increases in physical well-being. They expressed a variety of different ways they coped with everyday stressors. 
One member said, "A bottle of whisky, a bag of dope ... It makes me feel great," and another said meditation is one of the ways he deals with pain and stress. Many of the men discussed a wide range of coping mechanisms to deal with grief, loss, and low self-worth and self-esteem. Sharing coping methods can help members learn about different ways to deal with stress, and how that influences their physical health. Being involved in the DUDES Club appears to have led the men to take a variety of actions to improve their health. Some members indicated that they got blood tests and liver tests, followed through with suggested tests, and participated in preventative screening such as prostate exams during the annual DUDES Club Health Fair. Some attributed this improvement in health-seeking behaviours explicitly to the DUDES Club:

Member: You know what, I never had a prostate exam before I came to the DUDES Club, one year I wasn't going to do it, then the second health fair the doc said you're getting over 50 this time we'll check ... I actually went for a prostate exam. Now I do it every six months because there's no shame in it. I'm at the age where prostate is a serious thing.

Some members even brought substances in for identification:

Health care professional: a member of DUDES coming in and saying, "What is this?," before they take it, which is like, phenomenal. Because when I was using I didn't care what it was, you know? I've never tried that one before. And I mean, just, being conscientious enough to know that there's nurses in the building that they can go talk to. Which was awesome.

The men also talked about the importance of emotional, spiritual, and holistic health. In particular, many members discussed mental health as another issue they faced. Some members explained that they were first helped with mental health issues at the DUDES Club because they felt no other doctors wanted to help them. One member discussed other doctors as uncaring compared to the DUDES Club physician. However, mental health care can be much harder to obtain for low-income individuals, many of whom do not have access to a counsellor when they need one. Some members seemed to view the struggle of getting help as too much of a burden to bear:

For myself like if I were to deal with my mental health issues I've been battling for a couple of years. And if I was to deal with it I'd be fighting like a whole system right?

The DUDES Club in Vancouver temporarily provided a counsellor for the men and some members greatly appreciated the service:

When [the counsellor] first started it was rough. And my dad had died. Richard died. He carried me through it. And I'll tell you I was really rough. Fourteen people [died] in the next twelve and a half months, 17 people [died] in 15 [months]. I was done. [The counsellor] was good to have here.

Many of the members struggled with addictions, an issue intricately connected with historical and intergenerational trauma and continued systemic discrimination and violence. Through the Club, they safely discussed strategies and resources, often with a health care provider present to provide accurate information. Many of the men have friends who have died from substance use, and many have abstained from substance use for years. Many shared their stories and supported other members to heal from 
addictions. The sharing of stories of addiction with men at all different levels of substance use can help everyone to reconnect with their physical and mental health. The DUDES Club also helped the men to try to make their lives better despite addictions:

And if I'm going here all the time concentrating on the DUDES Club, I'm an addict too eh, I'm an alcoholic and an addict, it makes me not think about booze and alcohol, wanting to make my life better.

The DUDES Club offers a safe space where men can learn about health in an accessible way. The lowbarrier and non-hierarchical environment is unique for men impacted by structural and systemic violence. Many of the men have had negative experiences with standard health care, and the unique approach offered by the DUDES Club is particularly effective for both physical and mental health. The information the men learn in the Club propagates in the community as well, as the men share their knowledge and work to bring more men into the Club. The accessibility of health care is, of course, linked to constructions of masculinity.

\section{Disrupting Colonial Constructions of Masculinity}

The men in this study explained that they attended the DUDES Club to make friends, share their experiences and feelings, and seek health care-thus challenging colonial constructions of masculinity that paint men as strong, stoic, independent, dominant, and invincible. Many members also disrupted socially constructed gender norms by asserting the importance of family and creating a new family when they were estranged from their home communities.

Many of the men discussed how social constructions of masculinity appeared to prevent them from being mentally, physically, emotionally, and spiritually healthy. Some members expressed opinions that rigid notions of masculinity were a barrier to accessing health care, in line with the cultural stereotype that men do not go to the doctor: "I'm not going to get [a prostate exam], I'd rather die, forget it." In this case, death is described as preferable to a preventative screening procedure. Some of the members were explicitly aware of these stereotypes but appeared to still endorse them. Some of the anecdotes were more extreme; one provider indicated that if certain men had gone to the doctor, then they would still be alive.

The parents in the group pushed gender boundaries by talking about their roles as nurturers or primary caregivers. One member is a grandfather with sole custody of his grandson, who he brings to every DUDES Club meeting. Another member is a father, and he has brought his son to several meetings and is openly affectionate with him. One member even described how the Club helped him to know himself better as a father and a son. Further, when children are around, the rest of the men seem to want to be around them, perhaps because they crave the father-son relationship that they lack. One research team member brought her young son to a Vancouver DUDES Club meeting. He was treated so well by the members that after his initial hesitation at being there, by the end of the meeting he begged to come back to the next one. Being able to identify as a father and talk with other men about what that means is an important function of the DUDES Club. 
Lastly, the many discussions of the importance of men's only spaces revealed that these spaces enabled the deconstruction of colonial constructions of masculinity. Through this desire for men's only spaces, members seemingly voiced their need to connect with other men:

I'm going through a lot of rough stuff right now and I really wanted a men's group in this town.

Others talked about the lack of men's self-care opportunities compared to women. Another member explained how men's extracurricular activities problematically conform to masculine expectations:

But for men, we literally have nothing in terms of extracurricular activities or places where we can chill with other guys, there's nothing, where do you go? To the bar. Where do you go? To the park and drink, drink whatever rub, you know, just get in trouble.

Despite working to challenge colonial constructions of masculinity, there were potentially problematic discussions in some of the clubs. For example, in one focus group with club members, a member shared that he felt women now had it "easy" compared to men. However, in addition to modelling respectful behaviour toward women, men in leadership positions in the Club often redirected conversations to become learning moments. The Club's late co-founder, Richard Johnson, used to say, "We have ladies present," to cue club members to be respectful to the honorary "dudettes" who occasionally attended meetings. Other opportunities to change unhealthy norms around gender were created through guest speakers. For example, in 2015, a player from the BC Lions football team visited the Club to increase awareness and understanding about the impact of men's violence against women as part of the Be More Than a Bystander campaign - a groundbreaking initiative between the Ending Violence Association of BC (EVA BC, n.d.) and the BC Lions football team (n.d.).

Overall, members expressed how they strived for connection: to create family, to embrace fatherhood, and to build relationships with other men. In a way, the DUDES Club facilitates the men's push against traditional masculinity as part of their healing journey. However, patriarchy and colonial constructions of masculinity are only part of the systemic challenges members face.

\section{The Impact of Structural and Systemic Challenges on Members' Experiences}

At the core of many of the DUDES Club members' experiences, and intimately connected to themes discussed above, were structural and systemic challenges. Most members experienced a range of structural barriers including access to paid work and educational opportunities, decent food, nonjudgmental spaces, as well as ongoing experiences of trauma and racism. As one member at a northern pilot site described:

There's a lot of horrible racism in this town. Horrible, horrible racism. And that has to stop. We gotta change it, it's unsafe for any person of colour in this town. Doesn't matter whether you're First Nations or Black or Chinese or whatever. Part of that unfortunately is because of the systemic racism in the Canadian government, and the British Columbian government. And I can't do too much about the whole Canadian government, but I can make a change in my community. So, that's why I'm here. 
Structural barriers often prevented many members from seeking the social and emotional supports they needed as well as health services that could improve quality of life:

Member 1: I think the way the Downtown Eastside [of Vancouver] operates - and I think a lot of people would probably agree-when they talk, especially like the health care professionals, they don't listen to you. They hear you.

Member 2: They hear you but they think they know all the answers. They don't listen to what your real problems are. And I think that men get a better sense of the DUDES Club, that their actually, their real concerns are actually being heard by people.

These members' words speak to the power of belonging and ownership that they experienced at the DUDES Club in contrast to the barriers they experience every day. The DUDES Club is a space where they know they will be listened to and heard. It is a space of belonging that creates trust and willingness to engage with health structures as both patients and advocates:

Member: I'm a volunteer associated with here and the DUDES Club and all the other organizations like it actually puts you in a position where you're-like a delegate for, for your brothers on the street like they can approach you and you've got to connect right? And it's easier sometimes because a lot of people have issues dealing with health care, they're oppressed by the health care system and they can't, they just can't function to deal with health care professionals; they just shut down. And that's why advocates are necessary but sometimes advocates are difficult to obtain.

Persistent social and health inequities can have serious consequences for mental, physical, spiritual, and emotional well-being, making it a challenge to address health issues. The DUDES Club mitigates some of these stressors by providing a space where men can advocate for one another and feel that their voices will be heard.

Members frequently discussed housing as a structural and systemic challenge:

Okay so to get decent housing, I mean you're, you're better off to be homeless or in a shelter. Because if he's got a room, he's in SRO [single-room occupancy hotel], okay, he's got a roof, he's okay.

The SROs where many of the men live are often in deplorable conditions, and many men appeared to struggle with the idea that you have to be homeless or in crisis to get decent housing. Many participants discussed the lack of resources for men compared to women, and some described the restrictions that some housing providers place on overnight guests and visitors as more limiting than being in prison. For example, some members explained that shelters have check-in times that may interfere with work schedules and dehumanize patrons.

Finally, yet another health challenge that impacted many members was accessing food in neighbourhoods often characterized as "food deserts," geographic areas with a need for, but limited access to, healthy and affordable food options ( $\mathrm{Lu} \&$ Qiu, 2015): 
Down here food access is a hard thing. I'm HIV, some of us are, and you need to eat a proper diet. You don't. We eat a lot of crap because of what our budget is. We get good balance meals [at the DUDES Club]. Balance is a big thing for my health.

The DUDES Club is one place where the men know they will be nourished in all realms - physical, emotional, mental, and spiritual.

\section{Discussion}

In this article, we have presented results showcasing the impacts of the DUDES Club on its members. The DUDES Club facilitates feelings of brotherhood and community, produces tangible health benefits by providing accessible health information, challenges colonial constructions of masculinity, and addresses the structural and systemic challenges the members face. A strength of this work is the triangulation of the findings (Tracy, 2010), whereby members, Elders, providers, and health care professionals all discussed the benefits of the Club. We feel this is a strong assessment of the positive impacts of the DUDES Club.

The DUDES Club uniquely addressed members' health in multiple ways. The focus on social connection at the DUDES Club goes beyond a Western medical model of health, in which health care is conceptualized as a response to narrowly identified bio-medical problems. As many members discussed, they felt there was no place for men to go to connect with each other in their communities. The power of authentic social connections to impact health should not be underestimated. The founders saw the need for social connections and support for the many men who came to Vancouver Native Health because they were tired of sitting alone in their single-room occupancy hotels. Strong social support networks have been shown to reduce physiological responses to stress and, in communities with little social and emotional support, people are more likely to suffer from poor mental and physical health (Wilkinson \& Marmot, 2003). The DUDES Club also helped Indigenous men reconnect with their Indigenous culture, which can have potent impacts on well-being. Several studies have shown that a sense of belonging and connection to community are important aspects of urban Indigenous health and wellbeing (Cardinal, 2006; Environics Institute, 2010; Smylie et al., 2011). Furthermore, Indigenous connection to culture and community can buffer against the negative impacts of discrimination (e.g., Bombay et al., 2010; Spence et al., 2016). Culture is frequently the foundation of interventions designed by and for Indigenous Peoples (e.g., Fiedeldey-Van Dijk et al., 2017). Participant experiences in the DUDES Club confirmed the importance of a sense of belonging and connection to the community as key to (Indigenous and other) men's health and well-being.

The DUDES Club addresses men's health by providing accessible health information. Members described this as a unique part of the DUDES Club, as many members described how current and common health information practices are inaccessible. Men's groups are desperately needed to address men's health and well-being, including the high burden of health disparities experienced by men, such as death by suicide (Goldenberg, 2014; Kumar \& Tjepkema, 2019; Statistics Canada, 2019). Members identified low-barrier and non-hierarchical health care spaces as important to efforts to learn about and improve their health. This research demonstrated that the DUDES Club offers a safe and accessible space to access health information, which is crucial for men who experience ongoing structural and systemic violence within health settings. In our discussions with members, it was evident that 
participation in the DUDES Club helped them to better access health care, follow-through on medical appointments, and to better understand what they should do to take care of themselves. Doctors and nurses that we interviewed had similar conclusions. There also appears to be a ripple effect in which health information gained within the Club is disseminated in the community, which brings more men to the Club drawn by the promise of respectful and positive interactions. Multiple intersecting factors influence how and when men access health care services. Participants in this study clearly expressed the need for safe, accessible health care settings in which they feel their voice is truly heard.

Groups, such as the DUDES Club, may also help to address some of the systemic and structural challenges that men, and in particular Indigenous men, face. These challenges are inextricably linked to health. Many members discussed how the Club helped them navigate these systemic and structural challenges through access to advocates, another unique way the DUDES Club contributed to their health and well-being. For example, men and Indigenous people are much more likely to experience homelessness than women or non-Indigenous people in Canada (Gaetz et al., 2013). In some cities, Indigenous people make up $90 \%$ of the homeless population (CBC News, 2014), despite research showing safe, stable housing produces better long-term outcomes for vulnerable and marginalized populations (Goering et al., 2011). Homelessness has undeniable negative impacts on health (e.g., Guirguis-Younger et al., 2014). Indigenous men's groups like the DUDES Club may help members escape homelessness by providing help in navigating social service systems.

The DUDES Club also works to challenge the negative health impacts of toxic constructions of masculinity. Masculinity is essential to any discussion of a men's club. Some people are uncomfortable with the idea of a men's club and, indeed, people have expressed concerns at various community forums and online about how patriarchal structures continue to legitimize men's dominant position in society and justify unequal power relations between men, women, and people of different genders. What we have to say is this: Gender is a powerful determinant of health, shaping people's lives in many different ways. Too narrow of a focus on gender fails to capture the complexities embedded in these men's lives, such as the systemic and structural challenges that the members of this club face, including the impact of toxic definitions of masculinity. The findings of this study challenge socially constructed gender norms and call for a more nuanced understanding of Indigenous men's experiences of health and well-being. The existence of the DUDES Club, in and of itself, contributes to decolonizing notions of masculinity. Healthy Indigenous masculinities and communities require understanding how race and gender intersect to disadvantage Indigenous men and the associated negative ramifications for Indigenous communities (Innes \& Anderson, 2015). Decolonizing masculinity, to us, means supporting healthy ways for men to express masculinity to counter harmful paternalistic expressions of Western masculinity that differentially impact Indigenous men. This is not to say, of course, that male privilege does not exist, but to say that acknowledging the impact of patriarchy on people of all genders, and working to create healthier communities, will be for the betterment of everyone. Indeed, hegemonic constructions of masculinity may be part of why men's mental health has been largely overlooked by health authorities and policymakers and thus an inhibiting factor for men seeking health information (Seidler et al., 2016). Rather than considering systems of oppression and discrimination in isolation, our findings call for an intersectional understanding of gender in relation to other axes of oppression and how this shapes the needs, concerns, and priorities of particular groups of men (e.g., Collins, 2015). Acknowledging the intersectionality of gender does not devalue the rights of any particular gender-based or non-binary group but provides space to validate and understand diverse experiences. 
Though the DUDES Club has benefited those involved, it is not a cure-all. Disproportionate burdens of health on men - particularly Indigenous men - are based in deep-rooted colonial and patriarchal systems; one men's club cannot "solve" these issues. Furthermore, the DUDES Club in and of itself is not perfect. For example, some men have indicated they felt intimidated at the DUDES Club due to the size of the gathering. Clubs might work to include different formats to make all members comfortable. However, the DUDES Club is one very promising way to improve men's health and challenge colonial and patriarchal systems that exist to the detriment of people of all genders. Next, we turn to policy recommendations that may do the same.

\section{Policy Recommendations}

Evidence-based policy is paramount for feasible and tangible change to occur at the community level. In this section, we provide five policy recommendations for multiple levels of government, health care professionals, and communities interested in starting their own DUDES Club. Following some of the recommendations, we outline further policy considerations. There already exist many policy calls and recommendations for Indigenous health; as such, we connect some of our policy recommendations to concrete commitments already made in the Truth and Reconciliation Commission's (TRC, 2015) Calls to Action and the United Nations Declaration on the Rights of Indigenous Peoples (UNDRIP; United Nations, 2007).

\section{Honour Indigenous Practices and Traditions}

In keeping with the TRC's Calls to Action in Sections 18, 19, 20, 21, and particularly Section 22, Indigenous healing practices are to be recognized, valued, and used within the Canadian health care system, communities, and health and social service organizations, which can:

- Ensure club members are able to determine what Indigenous practices and traditions are appropriate for their social, cultural, and geographic context; and

- Provide funding for culturally appropriate supports and services that honour Indigenous healing practices.

\section{Policy Considerations}

For health care for Indigenous Peoples to be effective, it must be situated in Indigenous culture. This can mean many different things for different Indigenous Peoples. Readers should not mistake this as an indulgence of pan-Indianism. Appropriate cultural practices should be determined by the club members. In our case, the presence of Elders was fundamental. Members also talked about the healing power of nature and, since this data was collected, the DUDES Club has organized annual living-on-the-land retreats. Clubs also incorporate various cultural activities and ceremonies. Importantly, some of these cultural activities (like beading, drum-making, and sharing circles) challenge colonial constructions of masculinity. 


\section{Create a Sense of Ownership Over the Club}

In keeping with Article 3 (the right to self-determination) and Article 18 (rights to decision-making) of UNDRIP (United Nations, 2007), funders (including all levels of government), health care professionals, and community organizations can:

- Provide funding and support for DUDES Clubs that ensure members are able to determine activities, meals, health topics, and make other decisions about the club's function and purpose.

\section{Policy Considerations}

DUDES Clubs should not be another avenue for others to make decisions for the members. Many of the members live in intense manifestations of systemic and structural inequities that routinely strip them of their agency and autonomy. The sense of ownership that the members feel over the DUDES Club creates a sense of purpose and pride in the members and instills a sense of solidarity. Deciding on activities, meals, and health topics provides a sense of control. Lack of a sense of control can have tangible effects on health and well-being (e.g., Lachman et al., 2011). In this way, the DUDES Club may help to buffer the negative health ramifications of feeling powerless.

\section{Ground the Club in Social Connection}

Funders, health care providers, community organizations, and host communities can:

- Provide funding for Clubs that ensure space for social connections. For example, make sure that time for building relationships is built into the Club, whether as part of a simple social activity (e.g., playing bingo) or something more formal (e.g., a talking or sharing circle).

\section{Policy Considerations}

Club members, club providers, and health care workers all discussed the importance of social connections and bonding that the Club provides. Social support is linked to a variety of benefits, such as good mental health, positive affect, and lower rates of depression and PTSD symptomology (Lakey \& Orehek, 2011). As doctors often struggle to prescribe social support, providing space for creating relationships is a fundamental part of a functioning men's group. In the DUDES Club, relationships were created between the members, between the members and Elders, and between the members and the health care workers. This lack of hierarchy in the social support system was also important, as members see the doctor as another "DUDE," and thus feel a social connection with other members, regardless of their position. Ultimately, creating men's only spaces that are safe and trusted is a costeffective investment for the health care system by providing a model to successfully address upstream determinants of men's health.

\section{Take an Intersectional Approach to Health and Well-Being}

In keeping with the TRC's (2015) Call to Action 23 for cultural competency training for all health care professionals, all levels of government can work together to: 
- Provide funding to train health care professionals and other frontline workers involved with DUDES Clubs in culturally safe practices and how to connect with individuals deeply impacted by structural and systemic inequities; and

- Health care professionals involved in the Clubs should provide accessible health information.

\section{Policy Considerations}

Cultural safety training must be intersectional. It must include training to ensure providers and health care workers have a good understanding of the members' lived experiences to be able to provide space and information that is appropriate for the members' current lived realities, which are shaped by racism, socioeconomic circumstances, gender norms, and historical factors that continue to impact Indigenous Peoples today.

\section{Support for Indigenous-Driven Grassroots Initiatives Like the DUDES Club}

In keeping with UNDRIP Article 5 (affirming the right of Indigenous people to maintain and strengthen their own political, legal, economic, social, and cultural institutions; United Nations, 2007), all levels of government can work together to:

- Increase funding for grassroots initiatives grounded in Indigenous cultural practices, and support the work already being done to promote holistic healing in communities, particularly in the area of Indigenous men's health; and

- Provide funding for culturally responsive evaluation of grassroots, community, and Indigenous-led initiatives.

\section{Policy Recommendations}

The DUDES Club emphasizes Indigenous practices and traditions, ownership, social connection, and intersectional approaches. This results in somewhat uncommon funding costs. For example, it can be difficult to secure funding to pay Elders and counsellors, purchase food, and purchase materials for engaging in cultural activities. All of these components are fundamental to the success of the DUDES Club, and funders should consider them just as important as other associated costs.

\section{Conclusion}

The DUDES Club is an innovative model for addressing men's well-being, and physical, spiritual, emotional, and mental health. In the last five years, with the help of the First Nations Health Authority and Canadian Men's Health Foundation, the Club has expanded rapidly to dozens of locations across British Columbia. The model offers a unique way for men to engage with their health by providing a space where members can build brotherhood, challenge colonial constructions of masculinity, reconnect with their identities, access health care, and stand in solidarity to challenge structural and systemic barriers. Through holistic and non-judgmental approaches, the Club has successfully facilitated tangible health benefits for its members. It is an innovative model for policymakers to address upstream 
determinants of health, particularly socio-structural determinants, for the benefit of everyone in the community.

\section{References}

Assembly of First Nations, \& Health Canada. (2015). First Nations Mental Wellness Continuum Framework. https://thunderbirdpf.org/fnmwc-full-report/

Barton, A. (2016, July 23). In the Downtown Eastside, a brotherhood of support. The Globe and Mail. https://www.theglobeandmail.com/life/health-and-fitness/health/focus-dudesclub/article31082475/

Barker, B., Goodman, A., \& DeBeck, K. (2017). Reclaiming Indigenous identities: Culture as strength against suicide among Indigenous youth in Canada. Canadian Journal of Public Health, 108, e208-e210. https://doi.org/10.17269/cjph.108.5754

Battiste, M. (Ed.). (2009). Reclaiming Indigenous voice and vision. UBC Press.

BC Lions. (n.d.). Be more than a bystander. https://www.bclions.com/bemorethanabystander/

Bombay, A., Matheson, K., \& Anisman, H. (2010). Decomposing identity: Differential relationships between several aspects of ethnic identity and the negative effects of perceived discrimination among First Nations adults in Canada. Cultural Diversity and Ethnic Minority Psychology, 16, 507-516. https://doi.org/10.1037/a0021373

Cardinal, N. (2006). The exclusive city: Identifying, measuring, and drawing attention to Aboriginal and Indigenous experiences in an urban context. Cities, 23(3), 217-228. https://doi.org/10.1016/j.cities.2006.03.005

CBC News. (2014, March 28). Aboriginal homelessness an 'epidemic', York researcher says. CBC News. https://www.cbc.ca/news/canada/thunder-bay/aboriginal-homelessness-an-epidemicyork-researcher-says-1.2589861

Collins, P. H. (2015). Intersectionality's definitional dilemmas. Annual Review of Sociology, 41, 1-20. https://doi.org/10.1146/annurev-soc-073014-112142

Currie, C. L., Wild, T. C., Schopflocher, D. P., Laing, L., \& Veugelers, P. (2012). Racial discrimination experienced by Aboriginal university students in Canada. The Canadian Journal of Psychiatry, 57, 617-625. https://doi.org/10.1177/070674371205701006

DUDES Club. (n.d.). Our locations. https://www.dudesClub.ca/locations

Environics Institute. (2010). Urban Aboriginal Peoples study: Main report. https://www.uaps.ca/wp-content/uploads/2010/03/UAPS-Main-Report_Dec.pdf 
EVA BC. (n.d.). Ending Violence Association of BC. https://endingviolence.org/

Fiedeldey-Van Dijk, C., Rowan, M., Dell, C., Mushquash, C., Hopkins, C., Fornssler, B., \& Shea, B. (2017). Honoring Indigenous culture-as-intervention: Development and validity of the Native Wellness Assessment. Journal of Ethnicity in Substance Abuse, 16(2), 181-218. https://doi.org/ $\underline{10.1080 / 15332640.2015 .1119774}$

Gaetz, S., Donaldson, J., Richter, T., \& Gulliver, T. (2013). The State of homelessness in Canada 2013. https://www.homelesshub.ca/sites/default/files/attachments/SOHC2103.pdf

Goering, P. N., Streiner, D. L., Adair, C., Aubry, T., Barker, J., Distasio, J., Hwang, S. W., Komaroff, J., Latimer, E., Somers, J., \& Zabkiewicz, D. M. (2011). The At Home/Chez Soi Trial protocol: A pragmatic, multi-site, randomised controlled trial of a housing first intervention for homeless individuals with mental illness in five Canadian cities. BMJ Open, 1(2), 1-18. https://doi.org/10.1136/bmjopen-2011-000323

Goldenberg, S. L. (2014). Status of men's health in Canada. Canadian Urological Association Journal, $8(7-8)$, S142-144. https://doi.org/10.5489/cuaj.2308

Gone, J. P. (2013). Redressing First Nations historical trauma: Theorizing mechanisms for Indigenous culture as mental health treatment. Transcultural Psychiatry, 50, 683-706. https://doi.org/10.1177/1363461513487669

Gross, P., Efimoff, I., Lyana, P., Lambert, S., Joweski, V., Hau, K., \& Smye, V. (2016). The DUDES Club: A brotherhood for men's health. Canadian Family Physician, 62, e311-e318. https://www.cfp.ca/content/62/6/e311

Guirguis-Younger, M., McNeil, R., \& Hwang, S. W. (2014). Homelessness and health in Canada. University of Ottawa Press. https://doi.org/10.26530/OAPEN_515362

Hackett, L., Biderman, M., Doria, N., Courville, J., Bogner, E., Spencer, R., Miller, D., McMillan, J., \& Numer, M. (2021). A rapid review of Indigenous boys' and men's sexual health in Canada. Culture, Health \& Sexuality, 23(5), 705-721. https://doi.org/10.1080/13691058. $\underline{2020.1722856}$

Huang, T. (2019). 2019 Downtown Eastside local area profile. Simon Fraser University. https://www.sfu.ca/content/dam/sfu/continuing-studies/images/cityprogram/publications/2019\%20Downtown\%20Eastside\%20Local\%20Area\%20Profile.pdf

Heisler, M., Vijan, S., Makki, F., \& Piette, J. D. (2010). Diabetes control with reciprocal peer support versus nurse care management: A randomized trial. Annals of Internal Medicine, 153, 507-515. https://doi.org/10.7326/0003-4819-153-8-201010190-00007

Innes, R. A., \& Anderson, K. (Eds.). (2015). Indigenous men and masculinities: Legacies, identities, regeneration. University of Manitoba Press. 
Kumar, M. B., \& Tjepkema, M. (2019). Suicide among First Nations people, Métis and Inuit (20112016): Findings from the 2011 Canadian Census Health and Environment Cohort (CanCHEC). https://www150.statcan.gc.ca/n1/pub/99-011-x/99-011-x2019001-eng.htm

Lachman, M. E., Neupert, S. D., \& Agrigoroaei, S. (2011). The relevance of control beliefs for health and aging. In K. Warner Schaie \& S. L. Willis (Eds.), Handbook of the psychology of aging $\left(7^{\text {th }} \mathrm{ed}\right.$., pp. 175-190). Academic Press. https://doi.org/10.1016/B978-0-12-380882-0.00011-5

Lakey, B., \& Orehek, E. (2011). Relational regulation theory: A new approach to explain the link between perceived social support and mental health. Psychological Review, 118, 482-495. https://doi.org/10.1037/a0023477

Lu, W., \& Qiu, F. (2015). Do food deserts exist in Calgary, Canada? The Canadian Geographer, 59, 267282. https://doi.org/10.1111/cag.12176

Milligan, C., Neary, D., Payne, S., Hanratty, B., Irwin, P., \& Dowrick, C. (2016). Older men and social activity: A scoping review of Men's Sheds and other gendered interventions. Ageing \& Society, 36, 895-923. https://doi.org/10.1017/S0144686X14001524

Nelson, S. E., \& Wilson, K. (2017). The mental health of Indigenous Peoples in Canada: A critical review of research. Social Science \& Medicine, 176, 93-112. https://doi.org/10.1016/ j.socscimed.2017.01.021

Oliffe, J. L., Ogrodniczuk, J. S., Bottorff, J. L., Johnson, J. L., \& Hoyak, K. (2010). “You feel like you can't live anymore": Suicide from the perspectives of men who experience depression. Social Science \& Medicine, 74(4), 506-514. https://doi.org/10.1016/j.socscimed.2010.03.057

Pappas, S. (2019). APA issues first-ever guidelines for practice with men and boys. $A P A, 5 O(1), 34$. https://www.apa.org/monitor/2019/01/ce-corner

Patrick, C. (2014). Aboriginal homelessness in Canada: A literature review (The Homeless Hub Report Series Paper \#6). https://assets.documentcloud.org/documents/1098160/ aboriginalliteraturereview.pdf

Press, J. (2019, July 9). Nearly 50 per cent of Indigenous children in Canada live in poverty, study says. The Globe and Mail. https://www.theglobeandmail.com/canada/article-half-of-indigenouschildren-live-in-poverty-highest-rate-of-child/

Richmond, C. A. M., Ross, N. A., \& Egeland, G. M. (2007). Social support and thriving health: A new approach to understanding the health of Indigenous Canadians. American Journal of Public Health, 97. 1827-1833. https://doi.org/10.2105/AJPH.2006.096917

Robinson, M., Robertson, S., Steen, M., Raine, G., \& Day, R. (2015). Doing and rethinking: Building resilience with men. Mental Health Review Journal, 20, 185-198. https://doi.org/10.1108/ MHRJ-12-2014-0045 
Rotenberg, C. (2016). Aboriginal Peoples Survey, 2012 (Catalogue no. 89-653-X2016009). http://www.statcan.gc.ca/pub/89-653-x/89-653-x2016010-eng.pdf

Seidler, Z. E., Dawes, A. J., Rice, S. M., Oliffe, J. L., \& Dhillon, H. M. (2016). The role of masculinity in men's help-seeking for depression: A systematic review. Clinical Psychology Review, 49, 106118. https://doi.org/10.1016/j.cpr.2016.09.002

Smylie, J., Firestone, M., Cochran, L., Prince, C., Maracle, S., Morley, M., Mayo, S., Spiller, T., \& McPherson, B. (2011). Our health counts: Urban Aboriginal Health Database research project community report: First Nations adults and children City of Hamilton. De Dwa Da Dehs Nye's Aboriginal Health Centre, Ontario Federation of Indian Friendship Centres, Hamilton Executive Director's Aboriginal Coalition, and the Our Health Counts Governing Council.

Spence, N. D., Wells, S., Graham, K., \& George, J. (2016). Racial discrimination, cultural resilience, and stress. The Canadian Journal of Psychiatry, 61, 298-307. https://doi.org/10.1177/ $\underline{0706743716638653}$

Statistics Canada. (2019). Deaths and age-specific mortality rates, by selected grouped causes. https://www150.statcan.gc.ca/t1/tbl1/en/cv.action?pid=1310039201\#timeframe

Tannenbaum, C., \& Frank, B. (2011). Masculinity and health in late life men. American Journal of Men's Health, 5(3), 243-254. https://doi.org/10.1177/1557988310384609

Thorne, S. (2016). Interpretive description: Qualitative research for applied practice ( $\left.2^{\text {nd }} \mathrm{ed}.\right)$. Routledge. https://doi.org/10.4324/9781315545196

Tracy, S. J. (2010). Qualitative quality: Eight "big-tent” criteria for excellent qualitative research. Qualitative Inquiry, 16, 837-851. https://doi.org/10.1177/1077800410383121

Truth and Reconciliation Commission of Canada (TRC). (2015). Truth and Reconciliation Commission of Canada: Calls to Action. http://trc.ca/assets/pdf/Calls to Action English2.pdf

United Nations. (2007). United Nations Declaration on the Rights of Indigenous Peoples. http://www.un.org/development/desa/indigenouspeoples/wp-content/uploads/ sites/19/2018/11/UNDRIP_E_web.pdf

Wide, J., Mok, H., McKenna, M., \& Ogrodniczuk, J. S. (2011). Effect of gender socialization on the presentation of depression among men: A pilot study. Canadian Family Physician, 57, e74-e78. https://www.ncbi.nlm.nih.gov/pmc/articles/PMC3038836/

Wilkinson, R., \& Marmot, M. (Eds.). (2003). Social determinants of health: The solid facts $\left(2^{\text {nd }} e d.\right)$. World Health Organization.

Wilson, N. J., \& Cordier, R. (2013). A narrative review of Men's Sheds literature: Reducing social isolation and promoting men's health and well-being. Health and Social Care in the Community, 21(5), 451-463. https://doi.org/10.1111/hsc.12019 